\title{
Childhood sexual abuse as a potential predictor of psychotic like experiences in Tunisian college students
}

\author{
Fekih-Romdhane F, Tira S, Cheour M \\ Ibn Omrane Psychiatry Department; Razi Hospital
}

Background: Current research has shown that experiencing childhood trauma may predispose individuals for psychosis and expression of Psychotic like experiences (PLEs). The early detection of subjects at risk for psychotic disorders and the elucidation of factors related to the presence or exacerbation of PLEs may lead to the establishment of early intervention programs in a period of risk for the development of psychopathology as in adolescence/early adulthood. Systematic reviews and meta-analyses showed that individuals experiencing PLEs share multiple risk factors with those experiencing psychotic disorders (Linscott and Van Os, 2013), including stressful or traumatic events. We aimed to examine the prevalence and characteristics of PLEs in a sample of Tunisian college students, and to evaluate the impact of childhood traumas in the occurrence and severity of these experiences at the time of the survey.

Method: A total of 482 college students of three major universities of Tunis, namely, "Faculty of law and Political sciences of Tunis", "University of Human and Social Sciences of Tunis" and "National Engineering School of Tunis" (63.7\% female; aged 18-32) participated in a cross-sectional survey. All the students gathered at these universities, between February 1st and May 31st 2018, were invited to answer an anonymous, self-administered questionnaire. The Positive Subscale of Community Assessment of Psychotic Experiences, The Childhood Trauma Questionnaire, and the Depression Anxiety Stress Scales were used to assess childhood trauma, PLEs and depression, respectively.

Results: Among the positive items, those assessing Persecutory Ideation had the highest prevalence (93.6\%), followed by items measuring Magical Thinking (91.9\%). Bizarre Experiences and Perceptual Abnormalities were reported at least sometimes by $87.1 \%$ and $46.1 \%$ 'of the students, respectively. About half of the students $(53.5 \%)$ reported at least one positive PLE 'nearly always', and $40.9 \%$ reported at least one negative PLE with this frequency. Emotional neglect was the most commonly reported type of childhood trauma $(90.7 \%)$, followed by physical neglect $(84.9 \%)$ and sexual abuse (61.4\%). Pearson product moment correlations (matrix found in Table 1) indicated that previous traumatic experiences (particularly emotional abuse, physical abuse and sexual abuse) significantly and strongly correlated with PLEs in the overall sample $(p<0.001)$. After controlling for demographic variables (gender, age) and psychosocial factors (drug use, depression), sexual abuse significantly contributed to PLEs.
Table 1. Pearson correlations between CAPE dimensions and study variables

\begin{tabular}{|c|c|c|c|c|c|c|c|c|c|}
\hline & 1 & 2 & 3 & 4 & 5 & 6 & 7 & 8 & 9 \\
\hline 1- Positive dimension & - & & & & & & & & \\
\hline 2-Negative dimension & $0.557^{* *}$ & - & & & & & & & \\
\hline 3- Depressive dimension & $0.537^{* *}$ & $0.677^{*}$ & - & & & & & & \\
\hline 4- Emotional abuse & $0.290^{* *}$ & $0.403^{*+}$ & $0.435^{* *}$ & - & & & & & \\
\hline 5- Physical abuse & $0.297^{* *}$ & $0.212^{* *}$ & $0.243^{* *}$ & $0.615^{*+}$ & - & & & & \\
\hline 6- Sexual abuse & $0.415^{*+}$ & $0.303^{* *}$ & $0.284^{* *}$ & $0.551^{*+}$ & $0.423^{* *}$ & - & & & \\
\hline 7- Emotional neglect & $0.117^{*}$ & 0.075 & 0.083 & $0.288^{*}$ & $0.187^{* *}$ & $0.136^{*}$ & - & & \\
\hline 8- Physical neglect & $0.090^{*}$ & 0.003 & 0.018 & 0.073 & 0.058 & 0.046 & $0.244^{* *}$ & - & \\
\hline 9- Age & $-0,033$ & $-0,063$ & 0,014 & $-0,018$ & 0,036 & 0,001 & 0,008 & 0,017 & - \\
\hline 10- Depression (DASS-D) & $0.328^{* *}$ & $0.501^{* *}$ & $0.635^{* *}$ & $0,333^{*}$ & $0,204^{* *}$ & $0,256^{*}$ & $0,135^{*+}$ & $-0,067$ & 0.028 \\
\hline
\end{tabular}

'Significant at $p \leq 0.05$; **Significant at $p \leq 0.01 ; C A P E$ : Community Assessment of Psychic Experiences; DASS. Depression Anxiety Stress Scales.]

Discussion: Our results build on previous literature that primarily focused on global childhood traumatic experiences, and consistently indicated an association between a specific dimension of trauma, which is sexual abuse, and PLEs. Child sexual abuse is a substantial risk factor for a range of mental disorders in both childhood and adulthood, including affective, anxiety, substance abuse, and personality disorders (Cutajar et al., 2010a).

The evidence for an association between child sexual abuse and subsequently developing psychotic disorders, however, remains unresolved (Cutajar et al., 2010b). Very few studies focused on the link between child sexual abuse and PLEs. A representative cross-sectional survey in a large representative general population sample of England ( $n=7353$ ) found that sexual abuse before the age of 16 was strongly associated with psychosis, particularly if it involved non-consensual sexual intercourse (Bebbington et al., 2011).

Conclusion: Child sexual abuse victims should receive systematic and complete psychological screening during their adolescence and early adulthood including, among others, PLEs and psychotic symptoms. In addition to striving to develop interventions that can prevent/reduce child sexual abuse occurrence, interventions are needed to enhance psychological support and improve social integration of these children by reducing stigma attached to these experiences. These measures may facilitate access to psychological health services in schools and communities, especially in our context.

\section{Sources:}

Linscott, R.J., \& Van Os, J., 2013. An updated and conservative systematic review and metaanalysis of epidemiological evidence on psychotic experiences in children and adults: on the pathway from proneness to persistence to dimensional expression across mental disorders. Psychol. Med. 43(6), 1133-1149.

Cutajar, MC, Mullen, PE, Ogloff, JR, Thomas, SD, Wells, DL, \& Spataro, J., 2010a. Schizophrenia and other psychotic disorders in a cohort of sexually abused children. Arch. Gen. Psychiatry 67(11), 1114-1119.

Cutajar, M.C., Mullen, P.E., Ogloff, J.R., Thomas, S.D., Wells, D.L., \& Spataro, J., 2010b. Psychopathology in a large cohort of sexually abused children followed up to 43 years. Child Abuse Negl. 34(11), 813-822. Bebbington, P., Jonas, S., Kuipers, E., King, M., Cooper, C., Brugha, T., Meltzer, H., McManus, S., \& Jenkins, R., 2011. Childhood sexual abuse and psychosis: data from a cross-sectional national psychiatric survey in England. Br. J. Psychiatry 199, 29-37. 